\title{
Growth, body composition and metabolism at neonatal and adolescent life stages in low birth weight offspring
}

\author{
J. M. Wallace, R. P. Aitken, J. S. Milne, T. Bake and C. L. Adam \\ Rowett Institute of Nutrition and Health, University of Aberdeen, Aberdeen, AB21 9SB, UK
}

Human epidemiological studies have linked presumed fetal malnutrition leading to low birth weight with the subsequent development of metabolic syndrome. Using our established ovine model of late onset placental growth restriction, we assessed the impact of poor prenatal fetal nutrient supply on birth weight, growth, body composition and metabolism in both genders during neonatal and adolescent life.

Singleton-bearing adolescent dams were fed control $(\mathrm{C})$ or high $(2.25 \times \mathrm{C})$ nutrient intakes during pregnancy to induce normal or compromised placental size, respectively ${ }^{(1)}$. The resulting normal $(\mathrm{N}$; twelve male and twelve female) or placentally growth-restricted (PGR; nine male and sixteen female) lambs suckled their ad libitum fed mothers until weaning at 11 weeks of age. Thereafter, offspring were individually housed and had ad libitum access to the same nutrient dense complete diet. Lamb growth rates were measured throughout and body composition was determined by Dual energy X-ray absorptiometry at weaning and at $\sim 35$ weeks of age (adolescence). At 7 weeks of age, lambs were fasted for $3 \mathrm{~h}$ prior to an intravenous glucose tolerance test (GTT). Three baseline blood samples were collected and metabolite responses measured at $+5,10,15,20,30,45,60,90$ and 120 min relative to glucose administration $(0.25 \mathrm{~g} / \mathrm{kg}$ body weight). A second GTT was performed at $\sim 28$ weeks of age after an overnight fast $(0.5 \mathrm{~g}$ glucose $/ \mathrm{kg})$. Plasma was analysed for insulin, glucose, NEFA and glycerol.

PGR males and females were 35 and $49 \%$ lighter than $\mathrm{N}$ lambs at birth, respectively, and had reduced girth and height (all $P<0.001$ ). Fractional growth rate to weaning was elevated in PGR $v$. N groups $(P<0.001)$, was highest in PGR males $(P<0.03)$ and was inversely correlated with birth weight $(r=-0.887, P<0.001)$. Dual energy X-ray absorptiometry assessment of body composition at weaning revealed reduced bone mineral density $(P<0.02)$ and increased total fat $\%(P<0.001)$ in PGR compared with $\mathrm{N}$ lambs (bone mineral density; $0.57 \pm 0.014 v .0 .62 \pm 0.010 \mathrm{~g} / \mathrm{cm}$, and fat; $24.1 \pm 0.95 v .16 .6 \pm 0.77 \%$, no effect of gender). Fractional growth rate from weaning to adolescence remained higher and height remained lower in PGR $v$. N lambs $(P<0.001)$ and both were greater in males than females $(P<0.002)$. At 35 weeks, bone mineral density remained higher in PGR than $\mathrm{N}$ lambs $(0.73 \pm 0.014 v .0 .86 \pm 0.012 \mathrm{~g} / \mathrm{cm}, P<0.001)$ and females were fatter than males $(31.1 \pm 0.92$ v. $20.2 \pm 0.88 \%, P<0.001)$. Fasting plasma insulin, glucose, NEFA and glycerol were higher in PGR than $\mathrm{N}$ lambs $(P$ ranging from $<0.035$ to $<0.001)$ at both 7 and 28 weeks of age. Insulin secretion after GTT was higher in PGR $v$. $\mathrm{N}$ groups at both ages $(P<0.02)$ and was greatest in PGR males. NEFA and glycerol concentrations after GTT were similarly increased $(P<0.02)$ in PGR lambs at both ages. At 28 weeks, but not at 7 weeks, glucose area under the curve after GTT was higher in PGR than N lambs $(P<0.02)$, indicative of glucose intolerance.

Therefore, low birth weight offspring of both genders exhibit rapid postnatal catch-up growth and altered body composition in our animal model. This was associated with increased insulin secretion, glucose intolerance and altered indices of fat metabolism during neonatal to adolescent life, all of which may presage poor health in adult life.

1. Wallace et al. (2006) Placenta 27, Suppl. A, S61-S68. 